\title{
A Boy with X-Linked Inhibitor of Apoptosis Protein (XIAP) Deficiency as the Initial Presentation of Pure Red Cell Aplasia
}

\author{
Soo Yeun Sim ${ }^{1}$, Hye Yeon Choi ${ }^{1}$, Seung Bum Han ${ }^{1}$, Nack Gyun Chung', Bin Cho', MyungShin Kim² and Dae Chul Jeong \\ Departments of ${ }^{1}$ Pediatrics and ${ }^{2}$ Laboratory Medicine, Catholic Genetic Laboratory Center \\ College of Medicine, The Catholic University of Korea, Seoul, Korea
}

\begin{abstract}
$\mathrm{X}$-linked inhibitor of apoptosis protein (XIAP) deficiency is a rare immunodeficiency disorder. A 13-month-old boy was diagnosed with pure red cell aplasia (PRCA) and treated with steroid and cyclosporine. Bone marrow showed diminished erythroid precursors, and positive results for Parvovirus B19 and Cytomegalovirus (CMV) infection. A genetic study of Diamond-Blackfan anemia was negative. Five months later, he was admitted due to fever and lymphadenopathy. PCR showed still positive for the Parvovirus, CMV, and Epstein-Barr virus (EBV). He was diagnosed with diffuse large B-cell lymphoma that was positive for EBV. The patient received chemotherapy with R-CHOP, and achieved complete remission. Immunoglobulin (Ig) levels were within an age-matched normal range until the completion of chemotherapy. Subsequently, he was admitted nine times due to recurrent pneumonia and acute otitis media between two and eight years old. We reanalyzed the Ig levels and lymphocyte subsets: IgG, IgA, and IgM were $30 \mathrm{mg} / \mathrm{dL}$, below $1.0 \mathrm{mg} / \mathrm{dL}$, and $36 \mathrm{mg} / \mathrm{dL}$, respectively. Lymphocyte subsets showed nearly absent CD19 (+) cells, but T- and NK cell counts were within normal ranges. A genetic study showed a hemizygous inframe deletion mutation in exon 4 of the IAP gene without the $S H 2 D 1 A$ mutation, consistent with a XIAP deficiency. Therefore, he was diagnosed with XIAP deficiency. Genetic analysis of his mother, aunt and elder sister showed that they were carriers of the same gene mutation. He receives intravenous Ig regularly with follow-up, and still requires a low dose steroid to maintain hemoglobin level. We report XIAP deficiency as initial presentation of PRCA.
\end{abstract}

Key Words: X linked inhibitor of apoptosis protein, Pure red cell aplasia, Epstein-Barr virus, Immunodeficiency
pISSN 2233-5250 / eISSN 2233-4580 https://doi.org/10.15264/cpho.2021.28.2.84 Clin Pediatr Hematol Oncol 2021;28:84 88

Received on September 2, 2021 Revised on September 28, 2021 Accepted on October 4, 2021
Corresponding Author: Dae Chul Jeong

Department of Pediatrics, College of Medicine, The Catholic University of Korea, 222 Banpodae-ro, Seocho-gu, Seoul 01659, Korea

Tel: $+82-2-2258-6180$

Fax: +82-2-537-4544

E-mail: dcjeong@catholic.ac.kr ORCID ID: orcid.org/0000-0003-0934-817X

\section{Introduction}

$\mathrm{X}$-linked inhibitor of apoptosis protein $(X I A P)$ deficiency syndrome is a rare primary immunodeficiency with dysgammaglobulinemia [1,2]. Patients with XIAP show intractable inflammatory bowel disease (IBD) or extreme vulnerability to Epstein-Barr virus (EBV), which frequently developed hemophagocytic lymphohistiocy- tosis (HLH) [1]. XIAP, as a XIAP/BICRC4 mutation is classified as a type $2 \mathrm{X}$-linked lymphoproliferative disease (XLP), while $S H 2 D 1 A$ mutation is classified as a type 1 XLP [3]. XLP is an inherited primary immunodeficiency with an incidence of 1-2 per million males. The clinical triad manifestations of XLP show susceptibility to EBV with HLH, lymphoma, and dysgammaglobulinemia [3]. XIAP is classified as a type 2 XLP according to genetic mutations and some laboratory and clinical differences 
[3,4]. The XIAP gene is related to signaling by the nucleotide oligomerization domain receptor (NOD)-like pattern, which is an intracellular sensor of infection as an inflammasome, although the XIAP gene has an anti-apoptotic property [3-5].

Pure red cell aplasia (PRCA) has various etiologies, including congenital or acquired conditions such as a viral infection [6]. The acquired PRCA is related to an underlying disease or viral infection with parvovirus 19 [6]. EBV infection in the post-transplantation state or enzyme deficiency contributed to the development of the aplastic crisis [7,8]. Here, we report a case of XIAP deficiency as the initial presentation of PRCA.

\section{Case Report}

An 8-year-old boy was admitted nine times because of recurrent pneumonia and acute otitis media since the age of 3 years. He was diagnosed with PRCA at 13 months of age, which was controlled by repeated transfusions and prednisolone with cyclosporine. At diagnosis, a bone marrow study showed the arrest of erythroid lineage maturation. A viral study showed positive parvovirus B19 and cytomegalovirus (CMV) by serology and RQ-PCR. We did not have any genetic results for Diamond-Blackfan anemia. Five months later, he was admitted because of remittent fever with enlarged cervical lymph nodes that had progressed for 2 weeks. His laboratory data showed hemoglobin $7.7 \mathrm{~g} / \mathrm{dL}$, platelet 100,000/ $\mu \mathrm{L}$, ferritin $788 \mathrm{ng} / \mathrm{mL}$, fibrinogen $146 \mathrm{mg} / \mathrm{dL}$, and triglyceride $199 \mathrm{mg} / \mathrm{dL}$. PCR for virus showed persistent positivity of parvovirus B19 and CMV and was newly identified for EBV. We performed lymph node biopsy and PET-CT, the results of which were compatible with diffuse large B-cell lymphoma positive for EBV (Fig. 1). He received six courses of chemotherapy with rituximab, cyclophosphamide, doxorubicin, vincristine, and prednisolone. He achieved complete response, but still showed fluctuation of hemoglobin. Immunoglobulin levels were within the age-matched normal range until the completion of chemotherapy.

The patient was diagnosed with bronchopneumonia based on physical and radiologic findings. Viral studies were still positive for parvovirus B19, but negative for CMV and EBV. We evaluated immunoglobulin (Ig) level and lymphocyte subsets due to recurrent infection and rituximab-containing chemotherapy. The lymphocyte subset showed CD3 83.6\%, CD4 18.8\%, CD8 31.6\%, CD19 0.4\%, CD56 13.9\%, and CD3+CD56+ 8.9\%, while the Ig levels were IgG: $30 \mathrm{mg} / \mathrm{dL}$ (normal range: $870-1,700 \mathrm{mg} / \mathrm{dL}$ ), IgA: below $1.0 \mathrm{mg} / \mathrm{dL}$ (normal range: 110-401 mg/dL), IgM: $36 \mathrm{mg} / \mathrm{dL}$ (normal range: 33-190 mg/dL), and IgE: below $1.0 \mathrm{IU} / \mathrm{mL}$ (normal range: <52 IU/mL). We performed a genetic study of immunodeficiency based on EBV-associated lymphoma and repeated infection. A genetic study showed a hemizygous in-frame deletion mutation of c.1045_1047delGAG, p.(Glu350del), consistent
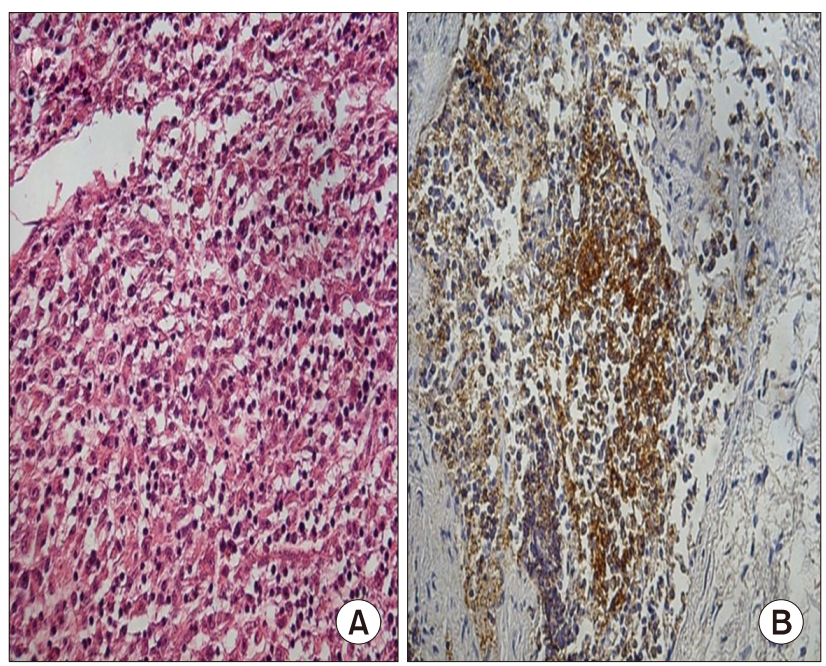

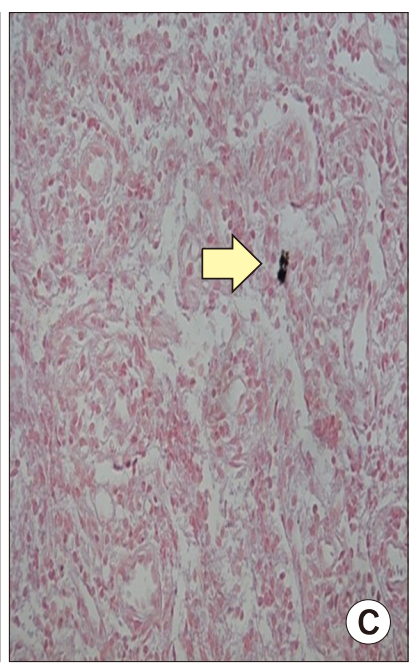

Fig. 1. Lymph nodes showed diffuse small lymphoblastic cells infiltration in Hematoxylin \& Eosin stain $(A, \times 100)$, positive CD20 cells in immunohistochemical stain (B), and positive Epstein-Barr virus (EBV) in situ hybridization (arrow) (C). These findings were compatible with diffuse large B-cell lymphoma presented with EBV. 
with XIAP deficiency (Fig. 2). A genetic study of family revealed his mother, aunt, and elder sister being carriers of the same gene mutation. He receives regular intravenous immunoglobulin due to dysgammaglobulinemia and low dose prednisolone to maintain his hemoglobin level. The patient did not experience infection after immunoglobulin replacement. Table 1 shows his clinical course with virus titer and immunoglobulin levels.

\section{Discussion}

Here, we present a case of XIAP deficiency with PRCA as the initial manifestation. The patient received transfusions with steroid and cyclosporine. He developed EBV -associated lymphoma after 5 months of PRCA treatment

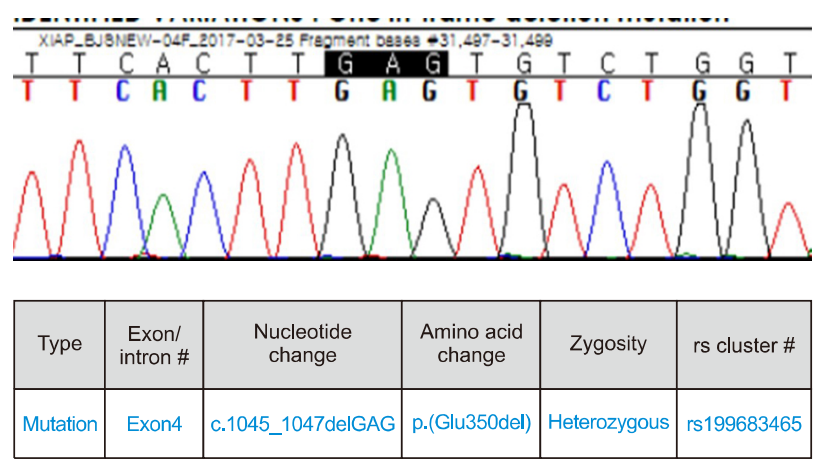

Fig. 2. This patient revealed the previously reported in-frame deletion mutation of XIAP gene on $\mathrm{Xq} 25$ by direct sequencing for exon 4 including intron-exon boundaries (reference sequence: NM_300635). later. The patient received chemotherapy with rituximab and achieved a complete response. The patient showed a normal range of Ig levels until completion of chemotherapy with rituximab, but Ig level decreased after each event of recurrent infection.

XIAP is composed of three baculovirus: the inhibitor of apoptosis domains, ubiquitin binding domain and C-terminal RING domain [3,5,9]. This protein has an anti-apoptotic activity which causes inhibition of programmed cell death and blocking of the activated state of caspase 3, 7, and 9 [5]. XIAP protein involves another signaling pathway or cellular response, including NFKB activation, MAPK pathway, TGF- $\beta$ receptor signaling, and autophagy [5]. XIAP also plays a role in innate immunity and the negative regulation of inflammation as a negative regulator of pro-inflammatory cytokine production and intracellular inflammasome activation [5,10].

In this patient, the initial finding was PRCA with an identification of the parvovirus and CMV. These findings may lead to excessive apoptosis of virus-infected erythroid cell lineage and overproduction of inflammatory cytokines to bone marrow precursors [3,5,10]. However, we could not identify the underlying disease at that time, although Diamond-Blackfan disease was ruled out through genetic study.

The patient was developed EBV-associated diffuse large B-cell lymphoma and received chemotherapy with rituximab and achieved complete response without re-

Table 1. The change of immunoglobulin levels and real time PCR results of Epstein-Barr virus (EBV) and cytomegalovirus (CMV) according to clinical course including chemotherapy with rituximab, recurrent infections, and low dose prednisolone with regular intravenous immunoglobulin

\begin{tabular}{|c|c|c|c|c|c|c|}
\hline \multirow{2}{*}{ Care for patient } & \multicolumn{2}{|c|}{ Chemotherapy with rituximab } & \multirow{2}{*}{ Post chemotherapy } & \multirow{2}{*}{$\downarrow \quad(\mathrm{IVIG})$} & \multirow{2}{*}{$\downarrow \quad(\mathrm{IVIG})$} & \multirow{2}{*}{$\downarrow \quad(\mathrm{IVIG})$} \\
\hline & $1^{\text {st }}$ cycle & $3^{\text {rd }}$ cycle & & & & \\
\hline Age (years old) & 1.6 & 1.9 & 4.2 & 8.3 & 8.5 & 9.5 \\
\hline EBV (copies/mL) & 176,772 & 5,589 & $\mathrm{~N} / \mathrm{E}$ & Not detected & & Not detected \\
\hline CMV (copies/mL) & 215,153 & 3,444 & $N / E$ & Not detected & & Not detected \\
\hline $\operatorname{lgG}(\mathrm{mg} / \mathrm{dL})$ & 889 & 575 & 135 & 30 & 300 & 474 \\
\hline $\operatorname{lgM}(\mathrm{mg} / \mathrm{dL})$ & 54 & 20 & $\mathrm{~N} / \mathrm{E}$ & 36 & 18 & 33 \\
\hline $\operatorname{lgA}(\mathrm{mg} / \mathrm{dL})$ & 45 & 33 & $\mathrm{~N} / \mathrm{E}$ & $<1.0$ & $<1.0$ & $<1.0$ \\
\hline Patient status & \multicolumn{2}{|c|}{ EBV associated lymphoma } & Recurrent Infections & \multicolumn{3}{|c|}{$\begin{array}{l}\text { Low dose prednisolone with IVIG infusion according } \\
\text { to IgG level }\end{array}$} \\
\hline
\end{tabular}

EBV, Epstein-Barr virus; CMV, cytomegalovirus; Ig, immunoglobulin; IVIG, intravenous immunoglobulin; N/E, not evaluated. 
lapse until the diagnosis of XIAP deficiency. The clinical manifestations of XIAP deficiency include high susceptibility to EBV infection, associated HLH, intractable IBD such as Crohn's disease, and without the development of lymphoma [4]. In type 1 XLP, EBV infection is associated with defects in the cytotoxicity of $\mathrm{CD} 8^{+} \mathrm{T}$ and NK cells $[3,4,11]$. In addition, the accumulation of apoptotic cells and persistence of EBV-infected cells could lead to abnormal inflammation and then progress to HLH $[3,5,11]$. EBV is a well-known oncogenic virus, that induced lymphoma via viral and molecular mechanisms in XIAP deficiency [11,12]. XIAP deficient patients are not developed to lymphoma in contrast to type 1 XLP [5]. In patients with XIAP deficiency, cellular defects might exaggerate T cell apoptosis following TCR-induced activation [11], and NKT cells showed low number with defective function to EBV [13]. This patient received cyclosporine and prednisolone for pure red cell aplasia. The long-term use of immunosuppressive agents might be contributed to develop EBV associated lymphoma associated with defective NKT cell function [14].

The Ig level was nearly within normal until the completion of chemotherapy with rituximab in this patient. After a complete response, he suffered from recurrent infections, including pneumonia and acute otitis media. Hypogammaglobulinemia with low B cells was also observed in the patient. This mechanism may develop due to EBV infection. He was diagnosed with EBV-associated lymphoma, and received rituximab, and experienced recurrent infections. In XIAP deficiency, EBV contributes to the accumulation of apoptotic cells, maintenance of EBV-infected B cells, but exacerbation of B-cell death, although NK and $\mathrm{T}$ cell cytotoxicity may be normal [3,5,11]. Rituximab eliminates EBV-infected B cells, but might strongly contribute to the loss of Ig production of Ig by B cells in this genetic background. Fortunately, the patient did not have gastrointestinal problems associated with IBD until now. In XIAP deficiency, IBD is generally severe due to the elevated level of pro-inflammatory cytokines caused by defects in the inflammasome pathway $[1,5]$. We will closely monitor the gastrointestinal manifestations.
The optimal treatment for XIAP deficiency depends on the patient's status. Patient with EBV-associated HLH might be treated using steroids with chemotherapy, rituximab, or alemtuzumab. Replacement of Ig may be supportive of dysgammaglobulinemia [1,3]. However, hematopoietic stem cell transplantation (HSCT) recommended for survival, which is related to a reduced intensity conditioning regimen and controlled HLH status [5,15].

Here, we present a case of XIAP deficiency as the initial clinical manifestation of PRCA. However, due to the patient's symptoms, another genetic disorders in PRCA without congenital hematologic disease may be considered.

\section{Conflict of Interest Statement}

The authors have no conflict of interest to declare.

\section{References}

1. Latour S, Aguilar C. XIAP deficiency syndrome in humans. Semin Cell Dev Biol 2015;39:115-23.

2. Marsh RA, Madden L, Kitchen BJ, et al. XIAP deficiency: a unique primary immunodeficiency best classified as X-linked familial hemophagocytic lymphohistiocytosis and not as X-linked lymphoproliferative disease. Blood 2010;116:1079-82.

3. Filipovich AH, Zhang K, Snow AL, Marsh RA. X-linked lymphoproliferative syndromes: brothers or distant cousins? Blood 2010;116:3398-408.

4. Pachlopnik Schmid J, Canioni D, Moshous D, et al. Clinical similarities and differences of patients with X-linked lymphoproliferative syndrome type 1 (XLP-1/SAP deficiency) versus type-2 (XLP-2/XIAP deficiency). Blood 2011;117:1522-9.

5. Aguilar C, Latour S. X-linked inhibitor of apoptosis protein deficiency: more than an $\mathrm{X}$-linked lymphoproliferative syndrome. J Clin Immunol 2015;35:331-8.

6. Means RT Jr. Pure red cell aplasia. Blood 2016;128:2504-9.

7. Le Voyer T, Boutboul D, Ledoux-Pilon A, et al. Late-onset EBV susceptibility and refractory pure red cell aplasia revealing DADA2. J Clin Immunol 2020;40:948-53.

8. Zhu K, Chen J, Chen S. Treatment of Epstein-Barr virus--associated lymphoproliferative disorder (EBV-PTLD) and pure red cell aplasia (PRCA) with Rituximab following unrelated cord blood transplantation: a case report and literature review. Hematology 2005;10:365-70.

9. Speckmann C, Lehmberg K, Albert $\mathrm{MH}$, et al. X-linked inhibitor of apoptosis (XIAP) deficiency: The spectrum of presenting manifestations beyond hemophagocytic lymphohistiocytosis. Clin Immunol 2013;149:133-41.

10. Yabal M, Müller N, Adler $\mathrm{H}$, et al. XIAP restricts TNF- and RIP3-dependent cell death and inflammasome activation. 
Cell Rep 2014;7:1796-808.

11. Tangye SG. Genetic susceptibility to EBV infection: insights from inborn errors of immunity. Hum Genet 2020;139:885901.

12. Ayee R, Ofori MEO, Wright E, Quaye O. Epstein Barr virus associated lymphomas and epithelia cancers in humans. J Cancer 2020;11:1737-50.

13. Rigaud S, Fondanèche MC, Lambert N, et al. XIAP deficiency in humans causes an X-linked lymphoproliferative syndrome.
Nature 2006;444:110-4

14. Tanner JE, Alfieri C. Interactions involving cyclosporine A, interleukin-6, and Epstein-Barr virus lead to the promotion of B-cell lymphoproliferative disease. Leuk Lymphoma 1996; 21:379-90.

15. Ono S, Okano T, Hoshino A, et al. Hematopoietic stem cell transplantation for XIAP deficiency in Japan. J Clin Immunol 2017;37:85-91. 